\title{
Interferometría óptica para medidas de traslación piezoeléctrica
}

\author{
Albert Reyna Ocas ${ }^{1}$, Ilich Contreras Verástegui ${ }^{1}$ y Whualkuer Lozano Bartra*1,2 \\ ${ }^{1}$ Laboratorio de Óptica No Lineal, Facultad de Ciencias Físicas, Universidad Nacional Mayor de San \\ Marcos, Ap. Postal 14-0149, Lima, Perú \\ ${ }^{2}$ Facultad de Ciencias Naturales y Matemática, Universidad Nacional del Callao, Bellavista, Callao, \\ Perú
}

Recibido 2 abril 2011 - Aceptado 28 junio 2011

\begin{abstract}
En este trabajo usamos un simple interferómetro de Michelson para caracterizar la deformación de un material piezoeléctrico en función del voltaje y la frecuencia aplicada. Se obtuvieron los parámetros de los factores de calidad del piezoeléctrico, tales como, la frecuencia de resonancia, la constante de amortiguamiento y el factor de mérito, modelando su comportamiento como un oscilador forzado.

Palabras claves: Interferómetro de Michelson, piezoelectricidad, oscilador forzado.
\end{abstract}

\section{Optical interferometry for piezoelectric translation measurements}

In this work we used a simple Michelson interferometer to characterize the deformation of a piezoelectric material in function of voltage and frequency. We obtained the piezoelectric quality factors parameters, such as, the resonance frequency, the damping constant and the quality factor by simulating its behaviour like a driven oscillator.

Keywords: Michelson interferometry, piezoelectricity, forced oscillator.

\section{Introducción}

Las técnicas de triangulación en los sistemas de tiempo de vuelo son métodos no coherentes para mediciones de distancia en el orden de los milímetros. Sin embargo, desde la invención del láser en 1960, se ha usado esta radiación de gran coherencia para mediciones de distancia en la escala nanométrica. Esta herramienta óptica como unidad fundamental de medida sigue siendo la base de la metrología óptica [1], disciplina que consiste de una gran variedad de técnicas de medición que permite caracterizar los desplazamientos de dispositivos de translación desde resolución micrométrica hasta nanométrica basados en el fenómeno de interferencia óptica. Mediciones rigurosas de gran resolución son exigidas y demandadas por la industria para la calibración de máquinas, herramientas, sistemas de topografía, robótica, etc. [2], por tanto, se han desarrollado varios métodos de medición, basados en la interferencia de radiaciones de múltiples longitudes de onda o interferencia de luz blanca, para realizar mediciones y verificaciones de distancias absolutas. La interferometría óptica se caracteriza por ser la herramienta más

\footnotetext{
*whualkuer@yahoo.com.br
}

versatil para realizar mediciones precisas sin perturbar las mismas por el contacto físico entre el aparato de medición y la muestra [3].

En este trabajo presentamos los resultados experimentales de mediciones de desplazamiento de un material piezoeléctrico en función de la frecuencia y voltaje aplicado y determinamos la frecuencia de resonancia y el factor de mérito del material usando un interferómetro básico de Michelson.

\section{Teoría}

Albert Abraham Michelson diseñó el interferómetro para medir conjuntamente con Edward Morley el movimiento del éter respecto al movimiento de la tierra [4]. Las medidas de la velocidad de la luz obtenidas con la extrema resolución de este instrumento permitió deducir la no existencia del éter. Desde entonces, este dispositivo se ha convertido en uno de los instrumentos más difundidos para las mediciones de desplazamientos muy pequeños. La implementación del láser dentro de este aparato hizo posible realizar mediciones precisas del orden de $\lambda / 2$. El fenómeno de interferencia produce 
un patrón de imágenes oscuras y brillantes a las que llamamos franjas de interferencia destructiva y constructiva, respectivamente, las cuales son el resultado de la superposición de dos frentes de onda $[5,6]$, donde las intensidades del campo eléctrico de las ondas reflejadas y transmitidas con frecuencias $\omega$ en un punto de interferencia $P$ se expresan como

$$
\begin{aligned}
& E_{1}=E_{0} \operatorname{sen}(\omega t), \\
& E_{2}=E_{0} \operatorname{sen}(\omega t+\phi),
\end{aligned}
$$

con una diferencia de fase $\phi$ que depende de la diferencia de camino óptico $\Delta=2 x(t)$ recorridas por las ondas reflejadas y transmitidas como se muestran en la Fig.(1), según la relación

$$
\phi=\frac{2 \pi}{\lambda} \Delta=\frac{2 \pi}{\lambda} 2 x(t),
$$

donde $\lambda$ es la longitud de onda del láser.

Debido a que el piezoeléctrico responde a una señal sinusoidal suministrada por un generador de funciones a una frecuencia de excitación $f_{0}$, el desplazamiento $x(t)$ del material piezoeléctrico, viene expresado por

$$
x(t)=A \operatorname{sen}\left(2 \pi f_{0} t\right),
$$

donde $A$ es la amplitud del desplazamiento. De acuerdo a las ecuaciones (1), (2) y al principio de superposición, la intensidad del campo eléctrico resultante en un punto $P$ está definido como

$$
E_{p}=2 E_{0} \cos \left(\frac{\phi}{2}\right) \operatorname{sen}\left(\omega t+\frac{\phi}{2}\right) .
$$

Debido a que la intensidad de una onda es proporcional al cuadrado de la amplitud resultante del campo eléctrico en el punto de interferencia, tenemos que

$$
I \propto E_{0}^{2}=4 E_{0}^{2} \cos ^{2}\left(\frac{\phi}{2}\right) \operatorname{sen}^{2}\left(\omega t+\frac{\phi}{2}\right) .
$$

Por lo tanto, usando las ecuaciones (3), (4) y (6) obtenemos la intensidad promedio en función del tiempo en el punto $P$,

$$
I(t)=I_{0} \cos ^{2}\left(\frac{2 \pi}{\lambda} A \operatorname{sen}\left(2 \pi f_{0} t\right)\right) .
$$

Posteriormente, para analizar la curva de resonancia del material piezoeléctrico, se recurre a un modelo mecánico de un oscilador armónico forzado con amortiguamiento cuyo desplazamiento se describe mediante la ecuación diferencial

$$
m \ddot{x}+m \gamma \dot{x}+m \omega_{0}^{2} x=F \cos (\omega t),
$$

donde $\omega_{0}$ es la frecuencia natural de oscilación del material piezoeléctrico, $\omega$ es la frecuencia de la señal inyectada, $m$ es la masa de la parte móvil del piezoeléctrico y $F$ es la fuerza que se ejerce sobre el mismo. Una solución particular de la Ec.(7) es dada también por la Ec.(3). Luego de un desarrollo matemático escribimos la amplitud de oscilación del material piezoeléctrico como

$$
A(\nu)=\frac{1}{4 \pi^{2}} \frac{F}{m} \frac{1}{\sqrt{\left(\nu_{0}^{2}-\nu^{2}\right)^{2}+\left(\frac{\nu \gamma}{2 \pi}\right)^{2}}},
$$

donde $\nu_{0}$ es la frecuencia de resonancia, $\Gamma=\gamma / 2 \pi$ es el coeficiente de amortiguamiento que define la calidad o agudeza de la curva de resonancia y $Q=\nu_{0} / 2 \Gamma$ es el factor de mérito. La razón $F / m$ define la amplitud de la curva Lorentziana.

\section{Resultados y discusión}

En la figura 1, se aprecia un esquema del interferómetro de Michelson. Los brazos usados en el experimento fueron de $15 \mathrm{~cm}$ de longitud aproximadamente. Usamos como fuente de luz, un láser de He-Ne de longitud de onda en $633 \mathrm{~nm}$ y $5.0 \mathrm{~mW}$ de potencia media. La luz del láser fue dividida por un divisor de haz 50:50 hacia los espejos recubiertos de plata en primera fase $M_{1}$ y $M_{2}$. El espejo $M_{1}$ es adherido al material piezoeléctrico que, también, está conectado a un generador de funciones Tektronix AFG310. La señal resultante debido a la interferencia de estos dos haces es llevada directamente a un detector rápido DET100A de la empresa Thorlabs el cual está conectado a un osciloscopio digital Tektronix TDS1002, y este a un computador personal para la adquisición de datos.

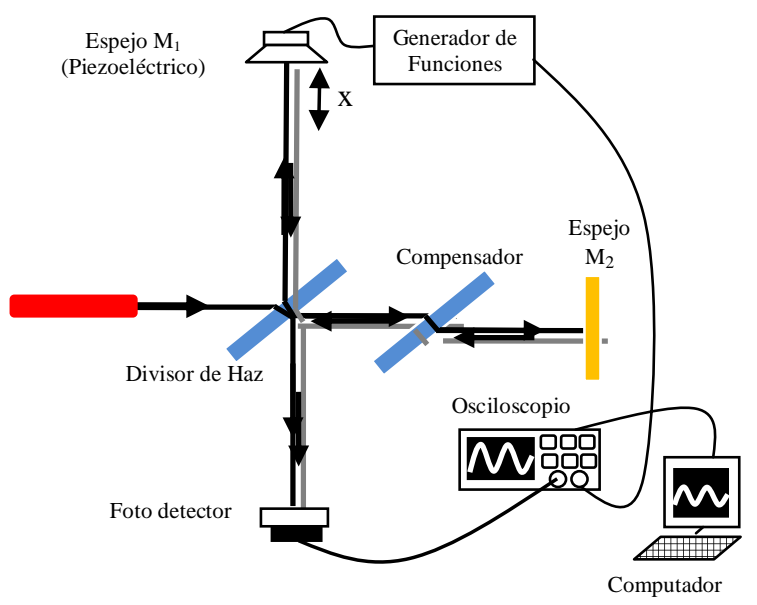

Figura 1: Esquema del interferómetro de Michelson.

La figura 2 muestra la traslación del material piezoeléctrico en función del voltaje aplicado, donde la línea roja punteada son los puntos mostrando las oscilaciones experimentales de las franjas de interferencia según el 
valor de la amplitud aplicada en el generador de funciones y la línea sólida azul es el ajuste teórico obtenido a partir de la Ec.(6).

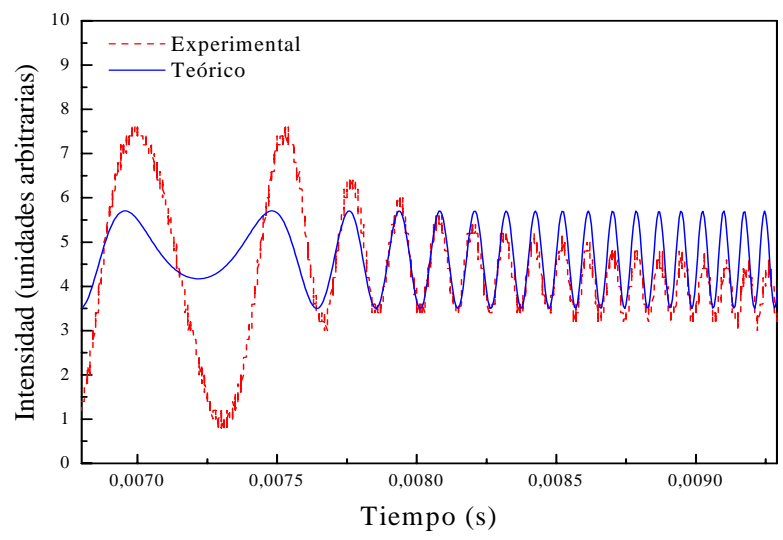

Figura 2: Las amplitudes de oscilación del material piezoeléctrico en función del tiempo.

En la figura 3, mostramos los desplazamientos que experimenta el material piezoeléctrico para voltajes de $0.3 \mathrm{~V}$ a $1.5 \mathrm{~V}$ para una frecuencia constante de 100 Hz. También realizamos medidas para diferentes valores de la frecuencia, pero manteniendola constante para el rango de voltajes aplicados 0.3-1.5 V. De esta forma, pudimos observar que los valores de las amplitudes de desplazamiento del material piezoeléctrico se comportan de manera lineal con respecto al voltaje suministrado, tal como se muestra en la Fig.4.

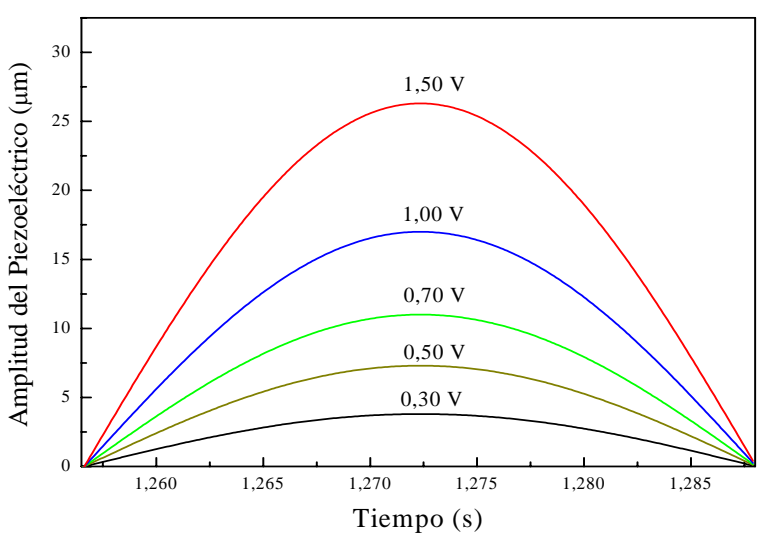

Figura 3: Desplazamientos del material piezoeléctrico desde $0.30 \mathrm{~V}$ hasta $1.5 \mathrm{~V}$ para una frecuencia constante de 100 $\mathrm{Hz}$.

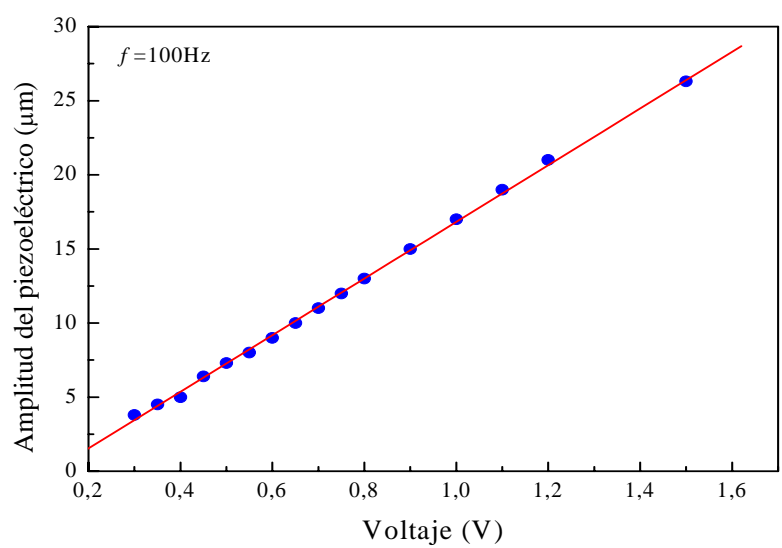

Figura 4: Dependencia lineal del desplazamiento del piezoeléctrico respecto al voltaje aplicado a la frecuencia de 100 $\mathrm{Hz}$.

Para obtener los puntos experimentales de la frecuencia de resonancia del material piezoeléctrico mostrados en azul, se realizaron mediciones variando la frecuencia en un rango de 10.0 a $100.0 \mathrm{~Hz}$, manteniendo constante la amplitud del generador de funciones para diferentes valores de frecuencia.

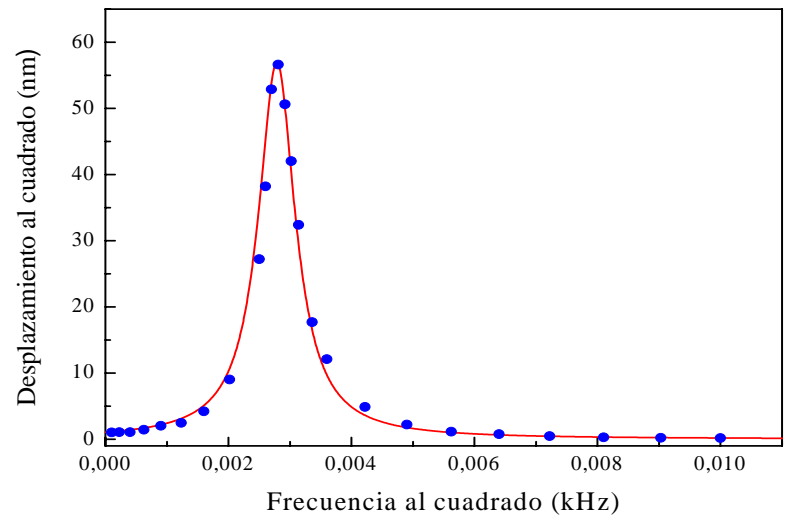

Figura 5: Determinación de la frecuencia natural del material piezoeléctrico.

En la Fig. 5, podemos observar que el valor experimental de la frecuencia de resonancia promedia es 53.0 Hz. Se observó que la amplitud de desplazamiento del material piezoeléctrico aumentaba cuando la frecuencia se aproximaba a la frecuencia de resonancia y disminuye cuando la frecuencia se alejaba de la frecuencia de resonancia. La línea roja sólida es el ajuste teórico de la función lorentziana obtenida a través de la Ec.(8), esta curva nos permite estimar el coeficiente de amortiguamiento $\Gamma$ y el factor de mérito $Q$ con valores de $100.0 \pm 2.0 \mathrm{~Hz}$ y $3.5 \pm 0.4$ del material piezoeléctrico, 
respectivamente. Para analizar el comportamiento de la amplitud del piezoelétrico con respecto a la frecuencia, realizamos mediciones de la frecuencia desde $10.0 \mathrm{~Hz}$ hasta $1.0 \mathrm{kHz}$, tal como se muestra en la Fig. 6.

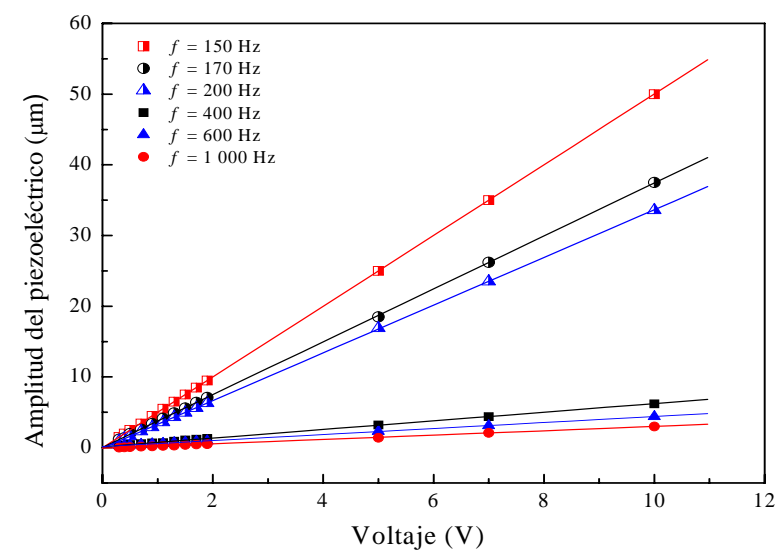

Figura 6: Respuesta lineal del material piezoeléctrico a diferentes frecuencias.

Aquí se observa que, para valores lejanos de la frecuencia de resonancia la amplitud de desplazamiento de material piezoeléctrico no varia significativamente. Por lo que inferimos que para valores mayores de $400 \mathrm{~Hz}$ la amplitud de desplazamiento del material piezoeléctrico es aproximadamente constante.

\section{Conclusiones}

La medición experimental de los valores de la amplitud de desplazamiento de un material piezoeléctrico han sido obtenidos para diferentes valores de voltaje y hemos observado que para frecuencias mayores a $400 \mathrm{~Hz}$ la amplitud de desplazamiento del material piezoeléctrico es constante. Observamos, también, que el valor de la frecuencia de resonancia y factor de mérito para un altavoz de 6 pulgadas puede ser simulado por el modelo del oscilador oscilador armónico forzado con amortiguamiento. Por su simplicidad, este experimento de enseñanza es adecuado para ser implementado en un curso de laboratorio de óptica moderna para estudiantes de pregrado.

\section{Agradecimientos}

Este trabajo fue parcialmente financiado por el Vicerrectorado Académico y por el Vicerrectorado de Investigación, a través del Consejo Superior de Investigación de la UNMSM.

\section{Referencias}

[1] Richard K. Leach, Fundamental Principles of Engineering Nanometrology, Elsevier, Amsterdam (2010).

[2] Hernán M. Miranda, Martín Coronel, Jorge R. Torga, Caracterización de desplazamientos en un sistema de translación por métodos ópticos. IV Conferencia Panamericana de Ensayos No Destructivos, Buenos Aires (2007).

[3] R. Rodríguez, J. Rayas, A. Martínez, A. Dávila. Algunas aplicaciones industriales de la interferometría electrónica de patrones de moteado. Departamento de Metrología Óptica, Centro de Investigaciones en Óptica, León, México y Centro de Investigación en Matemáticas, Guanajuato, México.

[4] A. Michelson, H. Lorentz, The Astrophysical Journal, LXVII, 5 (1928).

[5] María L. Calvo, Óptica Avanzada, Editorial Ariel Ciencia, Barcelona (2002).

[6] Eugene Hecht, Óptica, Addison Wesley Iberoamericana, Madrid (2000). 